\title{
Professional roles of female urologists: A webinar-based survey of perceptions and obstacles to career development
}

\author{
Sufyan Ibrahim ${ }^{1,2}$, Amelia Pietropaolo ${ }^{2,3}$, Nithesh Naik ${ }^{2,4}$, Anita Patel ${ }^{5}$, Milap J. Shah ${ }^{2,6}$, \\ Patricia Zondervan ${ }^{7}$, Jean McDonald ${ }^{8,9}$, BM Zeeshan Hameed ${ }^{2,6}$, Bhavan Prasad Rai ${ }^{2,10}$, \\ Hadis Karimi ${ }^{11}$, Bhaskar K. Somani ${ }^{2,3}$, Joanne Cresswell ${ }^{12}$ \\ ${ }^{1}$ Kasturba Medical College Manipal, Manipal Academy of Higher Education, Manipal, India; \\ 2 i-TRUE (International Training and Research in Uro-oncology and Endourology) Group; \\ ${ }^{3}$ Department of Urology, University Hospital Southampton NHS Trust, Southampton, UK; \\ ${ }^{4}$ Faculty of Engineering, Manipal Institute of Technology, Manipal Academy of Higher Education, Manipal, India; \\ ${ }^{5}$ Global Hospitals, Maharashtra, India; \\ ${ }^{6}$ Department of Urology, Kasturba Medical College Manipal, Manipal Academy of Higher Education, Manipal, India; \\ ${ }^{7}$ Academic Medical Center, University of Amsterdam, Netherlands; \\ ${ }^{8}$ North Middlesex University Hospital, Sterling Way, London, United Kingdom \\ ${ }^{9}$ Weymouth Street Hospital, Marylebone, London, United Kingdom; \\ ${ }^{10}$ Department of Urology, Freeman Hospital, Newcastle upon Tyne NE7 7DN, United Kingdom; \\ ${ }^{11}$ Manipal College of Pharmacy, Manipal Academy of Higher Education, Manipal, India; \\ 12 The James Cook University Hospital, South Tees Trust, Middlesbrough, United Kingdom.
}

\begin{abstract}
Summary Background: Urology, traditionally a maledominated specialty, keeping pace with the quickly changing gender landscape, has been characterized by waves of feminization. This study aims to understand the perspectives of women urologists on the obstacles to their career development, and the impact of such hurdles on their professional roles in urological education, practice, and leadership. Methods: 119 female urology residents/consultants were surveyed via a webinar-based platform, covering relevant questions on domains of Academia, Mentorship, Leadership, Parenting, and Charity. Statistical analysis was done using frequency distribution based on the responses.

Results: $46.8 \%$ of the respondents felt that there is an under-representation of women in academia. 'Having a good mentor' was the most important factor for a novice to succeed in academia (68\%). The most important trait in becoming a good leader was 'good communication skills' (35\%), followed by 'visionary' (20\%). The greatest challenge faced by leaders in the medical field was considered as 'time management' (31.9\%). Only 21.2\% of the participants felt difficulty in having a work-personal life balance, whereas $63.8 \%$ of them found it difficult only 'sometimes'. As a working parent, 'the guilt that they are not available all the time' was considered the most difficult aspect (59.5\%), and 'more flexible schedule' was needed to make their lives as a working parent easier (46.8\%). 34\% of the respondents were affiliated with some charitable organizations. The biggest drive to do charity was their satisfaction with a noble cause (72.3\%).

Conclusions: Need for increased encouragement and recruitment of females into urology, and to support and nurture them in their career aspirations.
\end{abstract}

KeY WORDS: Leadership; Mentorship; Academia; Parenting; Charity; Women in Urology; Gender disparity.

Submitted 17 October 2021; Accepted 26 October 2021

\section{INTRODUCTION}

The year 1962 recognized the first woman as a board-certified urologist in the United States (1). Urology, like most other surgical disciplines of medicine, has traditionally been a male-dominated specialty. However, keeping pace with the quickly changing gender landscape, medical professions are also characterized by waves of feminization, with a steady and significant increase in the number of women working in urological practice and research over the recent years (2). Although still a minority, female urologists tend to perform more gender-neutral index surgical procedures on female patients relative to their male counterparts (3). Despite more women being attracted towards medicine, and qualifying as doctors, their career progression can be hindered by organizational barriers with rigid career structures that may favor their male counterparts (4). Continued advancement of women in academic surgery is dependent on addressing these concerns including the lack of gender equality, effective mentorship, and work-life balance facilitating family responsibilities (5). The rising trends of invited female speakers at academic conferences present an opportunity for increased representation within urology leadership (6).

We aimed to understand the perspectives of women urologists on the obstacles to their career development, and the impact of such hurdles on their professional roles in urological education, practice, and leadership, through a webinar-based survey. Our objective was to understand the different barriers to career development experienced by female urologists, the gender disparity in urology, and the common factors affecting them. We also aimed to acknowledge the interest and involvement of female urologists in mentorship, leadership, and charity-based services.

No conflict of interest declared. 


\section{Methods}

A webinar titled "Today's Women - Tomorrow's Leaders: Urology Leading The Way" was conducted through an online platform with leading female urologists from India, United Kingdom, and the Netherlands as expert panelists. The target study population and potential respondents were female residents (urology trainees, registrars, and fellows) and urologists (consultants and practicing urologists). The webinar was attended by a total of 659 participants. A total of 43 different countries were represented by one or more participants in the webinar. Out of the total number of participants attending the webinar, 65\% ( $n=428)$ identified themselves as female participants, of which $28 \%(n=119)$ were female consultants or resident urologists, the responses of whom are included in this study. It is of interest that $35 \%$ of participants were male, and this may reflect increasing interest to make Urology a more inclusive specialty. A structured poll-based survey to investigate the female-related obstacles was conducted simultaneously with the webinar in progress. The five cornerstone topics that were discussed by the eminent speakers in the webinar were under the aegis of (a) Academia, (b) Mentorship, (c) Leadership, (d) Parenting, and (e) Charity in Urology. The surveying content was initially drafted by the investigating team, which was then circulated and reviewed by the i-TRUE working group. The study was approved by the Survey and Behavioural Institutional Ethics Committee (IEC) of Kasturba Medical College, Manipal (Reference No: 628-2020). This methodology is a relatively unique way of receiving responses and has not been widely used in previous surveys. The introduction specifying the objectives and target audience of the study were informed by the moderators of the session. Implied consent was assumed when the respondent proceeded to the registration and completion of the webinar. The survey was anonymous. Internet Protocol (IP) restrictions were implemented, so one IP address could only complete the survey once. All the data were collected and were accessible only by the study investigators. Statistical data analysis was done using SPSS (version-26) software. Categorical data were presented with counts and percentages. Graphs and charts were appropriately plotted.

\section{RESULTS}

A total of 11 different questions related to the five domains were asked during the webinar. A single best response was considered for every question by every poll- respondent. Following are the results of responses from female urologists as mentioned in our inclusion criteria $(\mathrm{n}=119)$ with their graphical representation in Figure 1 and Figure 2.

The respondents felt that there is an under-representation of women in academia (46.8\%), while 38.3\% felt otherwise and $14.89 \%$ chose rather not to comment. According to the respondents, the most important factor for a novice to understand and succeed in academia was a good mentor' (68\%). The other traits that were considered important by others were 'a natural flair for research' (19.1\%), 'good project' (8.5\%) and 'being good at statistics' (4.2\%); surprisingly 'being a good writer' (0\%) was totally out of contention by respondents. A large fraction of our audience (44.6\%) had a fixed or structured training module/program at their hospital/institute, but $27.6 \%$ did not have it and the other $27.6 \%$ had unstructured training modules.

According to poll-respondents, the most important trait in becoming a good leader was 'good communication skills' (35\%), followed by 'visionary' (20\%). Remarkably, an equal number of respondents (15\%) considered the 'ability to prioritize and focus', 'influencer/empowering', and 'patience or good listener' as the most important qualities. The greatest challenge faced by leaders in the medical field was answered by a majority as 'time management' (31.9\%), followed by 'lack of resources' and 'coordination of work amongst members' (25.5\% each), 'assigning a task as per the ability of the worker or resident' (10.6\%) and 'patient mistrust' (6.3\%). An important and very pertinent issue highlighted during our webinar was that of a working parent. When polled if they have difficulty having a workpersonal life balance, $21.2 \%$ responded as 'yes' and 14.8\% checked 'no'. However, most of the respondents (63.8\%) found it difficult to balance their work and family only 'sometimes'. According to the respondents, the most difficult aspect that they consider as a working parent is that of 'the guilt that they are not available all the time' (59.5\%), while others thought 'lack of time with kids' (19.1\%), 'lack of time with spouse' (14.8\%) and 'unsupportive work colleagues' $(2.1 \%)$ as their important hurdles. Positively, a small (4.2\%) number of them believed that parenting and working are easy. 'More flexible schedule' was felt to be the most important factor that could make their lives as a working parent easier or better, by almost half of the respondents (46.8\%). Others considered 'more supportive boss or co-worker' (19.1\%), 'more meaningful work' (14.8\%), 'telecommuting' (12.7\%), and 'more help with chores or kids' (6.3\%) as the important factors for the same question. A total of $34 \%$ of the respondents were affiliated with some charitable organizations, a minor fraction (14.8\%) were dubious with their response, and 51\% were not associated with any. Of all the promoting factors, the biggest drive and

\section{Figure 1.}

The responses for trichotomous questions of the survey. 
Figure 2.

Representation of the responses by the participants $(n=119)$ to the questions on (a) Leadership (b) Parenting (c) Academia, Mentorship and Charity.

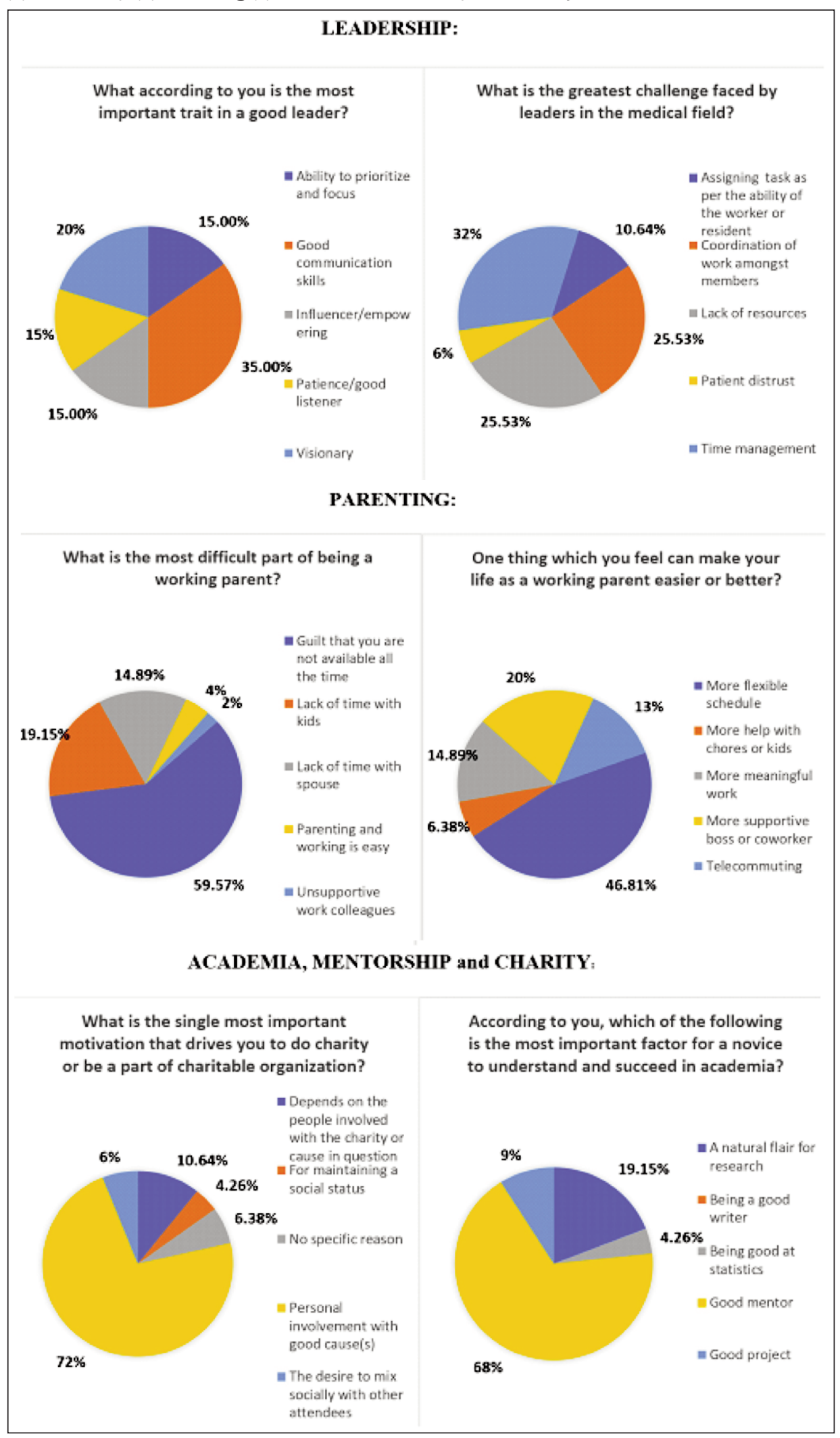

The people involved with the charity or cause in question was also a factor considered by many (10.6\%). The desire to mix socially with other attendees (6.3\%), no specified reason $(6.3 \%)$, and for maintaining a social status (4.2\%) were the lowly rated attributes for the given question.

\section{Discussion}

While the discordant representation of women in urology is well-established, only a few studies have attempted to delineate the cause of this disparity and the confounding effects it has on their personal lives (7). To our knowledge, there are no studies available in the current literature which address the perception of women urologists spanning across a full circle of academia, mentorship, leadership, parenting, and charity simultaneously.

Although the majority of our poll-respondents agree to the existence of under-representation of females in urology, $38.3 \%$ however, felt that this was not so significant. This may be partly explained due to an increasing prevalence of female urologists in the recent past accounting for about $9.2 \%$ of the total workforce and about $21 \%$ of the under-45-year-olds in countries like the USA (6). Such demographic trends have greatly improved the visibility of female urologists in developed countries, who are also at a greater likelihood of pursuing sub-specializations, and positively increasing their identification with leadership roles (8). However, a recent study has shown that despite specialization or seniority, female surgeons perform less complex cases than their male peers, hence more needs to be done to recruit

important source of motivation for our respondents to do charity or be involved as a part of a charitable organization, was their satisfaction with a noble cause (72.3\%). and retain all under-represented minorities, as greater diversity helps improve the outcomes of the general population (9). There is no single 'best approach' involved in 
training and navigating across academia, but the presence of mentors can foster a sense of professional identity and facilitate a novice experience through guidance, support, and encouragement (10). Most of our participants (68\%) felt that having a mentor, as a wise and trusted counselor, followed by a natural flair towards research are the keys to academic excellence. A healthy and strong mentormentee relationship stems based on consideration, great camaraderie, existing features of commonality, and virtues of confidentiality (11). Leaders are people with the capability to explicitly articulate protocols, augment productivity, motivate team members to achieve the desired goal to create a sustainable change in any profession (12). The core traits of becoming a good leader-talent, drive, willpower, practical wisdom, loyalty, ethical behavior, emotional intelligence, integrity, self-awareness, and resilience are all very essential (13). Our participants felt that exquisite communication skills are the most important attribute and a clear favorite prerequisite for charismatic leadership. This is in line with the expectations as interpersonal communication is the binding factor to show a leader's assuredness, decision-making skills, and the ability to convey the strategies and outcomes of the vision and the mission to the team (14). The circumstances are no different for leaders in medicine who aim to improve the quality of healthcare (15). The main challenges, according to our poll-respondents, were the effective management of time, lack of resources, and coordination of work amongst members. This is indeed in conjunction with the other studies on leadership where proactive and focused personal time management was identified to be necessary for optimizing organizational productivity (16). This also highlights the persistent issue of imbalance in the allocation of resources for utilization despite being the central function of healthcare delivery systems (14). Appropriate delegation of duties matching the amount of responsibility with capability and authority, with regular feedback, will help in empowering colleagues to reach their potential (17). Work-personal life harmony is particular to every individual and thus attainment of a 'balance', if it exists, is a very subjective feeling, explaining the reluctance of poll-respondents in answering the question (18). It continues to be a very challenging issue, as according to the National Physician Burnout and Suicide Report 2020, urology topped the chart with $54 \%$ of practicing urologists reporting that they are victims of physician burnout (19). The prime reason for this was due to the administrative burden with too many bureaucratic tasks to fulfill, as driven by workplace and organizational culture. Spending too many hours at work and lack of respect from colleagues were the other important reasons cited by many (19).

This is in concordance with our results as participants felt that the guilt of not being available at all times was their biggest concern. Parenting as female physicians may have additional challenges to address as they generally remain responsible for childcare and domestic activities compared to male counterparts, thus limiting their career advancement, while also feeling inadequate in performing the dual role (20). Strategies need to be implemented while structuring the programs to help strike a synergy in their personal and professional lives (19). Greater flexi- bility in the schedules, with allowance for dedicated maternity leaves for a longer duration, and support from employers and co-workers are the important contributors in this regard, as also evident from our survey (20).

\section{Conclusions}

Women play a vital role in all aspects of charity, leadership, academia, mentoring, and parenting. While urology is leading the way with more females taking up the specialty, gender disparity and traditional dogma is still a hindrance to it. Although urological societies recognize this, more needs to be done to encourage female recruitment into urology and to support and nurture them in their career pathways.

\section{FUNDING}

The authors report no involvement in the research by the sponsor that could have influenced the outcome of this work.

\section{AUthors' CONTRIBUtIONS}

Sufyan Ibrahim and BM Zeeshan Hameed have given substantial contributions to the conception or the design of the manuscript. Nithesh Naik, Amelia Pietropaolo, Milap Shah, Patricia Zondervan and Anita Patel to acquisition, analysis and interpretation of the data. All authors have participated to drafting the manuscript, Bhaskar K Somani, Bhavan Prasad Rai and Joanne Cresswell revised it critically. All authors read and approved the final version of the manuscript.

\section{ACKNOWLEDGEMENTS}

The authors acknowledge Kasturba Manipal College, Manipal for permitting to carry out the study.

\section{REFERENCES}

1. Rickey LM, Yang CC, Lamb DJ. Women in urology 2014. Urol Pract. 2014; 1:104-6.

2. Amir H, Beri A, Yechiely R, et al. Do urology male patients prefer same-gender urologist? Am J Mens Health 2018; 12:1379-83.

3. Oberlin DT, Vo AX, Bachrach L, Flury SC. The gender divide: the impact of surgeon gender on surgical practice patterns in urology. $J$ Urol. 2016; 196:1522-6.

4. Hirayama M, Fernando S. Organisational barriers to and facilitators for female surgeons' career progression: a systematic review. J R Soc Med. 2018; 111:324-34.

5. Seemann NM, Webster F, Holden HA, et al. Women in academic surgery: Why is the playing field still not level? Am J Surg. 2016; 211:343-9.

6. Capella C, Schlegel L, Shenot P, Murphy A. Female representation at high-profile urology conferences, 2014-2019: a leadership metric. Urology. 2021; 150:72-76.

7. Carr PL, Gunn CM, Kaplan SA, et al. Inadequate progress for women in academic medicine: findings from the National Faculty study. J Womens Health (Larchmt). 2015; 24:190-9.

8. Nettey OS, Fuchs JS, Kielb SJ, Schaeffer EM. Gender Representation in Urologic Subspecialties. Urology. 2018; 114:66-70.

9. Dai JC, Agochukwu-Mmonu N, Hittelman AB. Strategies for 
attracting women and underrepresented minorities in Urology. Curr Urol Rep. 2019; 20:61.

10. Kotsis SV, Chung KC. Application of the "see one, do one, teach one" concept in surgical training. Plast Reconstr Surg. 2013; 131:1194-1201.

11. Eby LT, Allen TD, Evans SC, et al. Does mentoring matter? A multidisciplinary meta-analysis comparing mentored and non-mentored individuals. J Vocat Behav. 2008; 72:254-267.

12. Vender RJ. Leadership: an overview. Am J Gastroenterol. 2015; 110:362-7.

13. Chan Z, Bruxer A, Lee J, et al. What makes a leader: Identifying the strengths of Canadian physical therapists. Physiother Canada. 2015; 67:341-8

14. de Vries RE, Bakker-Pieper A, Oostenveld W. Leadership = communication? The relations of leaders' communication styles with leadership styles, knowledge sharing and leadership outcomes. J Bus Psychol. 2010; 25:367-80.

15. Berghout MA, Fabbricotti IN, Buljac-Samardžic M, Hilders
CGJM. Medical leaders or masters? - A systematic review of medical leadership in hospital settings. PLoS One. 2017; 12:e0184522.

16. Kumar S, Adhish VS, Chauhan A. Managing self for leadership. Indian J Community Med. 2014; 39:138-42.

17. Zhang X, Qian J, Wang B, et al. Leaders' behaviors matter: The role of delegation in promoting employees' feedback-seeking behavior. Front Psychol. 2017; 8:920.

18. Dyrbye LN, Freischlag J, Kaups KL, et al. Work-home conflicts have a substantial impact on career decisions that affect the adequacy of the surgical workforce. Arch Surg. 2012; 147:933-9.

19. Medscape National Physician Burnout \& Suicide Report 2020: The Generational Divide (Internet). (cited 2020 Sep 11). Available from: https://www.medscape.com/slideshow/2020-lifestyle-burnout6012460\#1

20. Parsons WL, Duke PS, Snow P, Edwards A. Physicians as parents: Parenting experiences of physicians in Newfoundland and Labrador. Can Fam Physician. 2009; 55:808.

\section{Correspondence}

Sufyan Ibrahim, MD

Kasturba Medical College Manipal, Manipal Academy of Higher Education, Manipal (India)

Amelia Pietropaolo, MD

Bhaskar K Somani, MD

Department of Urology, University Hospital Southampton NHS Trust, Southampton (UK)

Nithesh Naik, MD

Faculty of Engineering, Manipal Institute of Technology, Manipal Academy

of Higher Education, Manipal (India)

Anita Patel, MD

Global Hospitals, Maharashtra (India)

Milap J Shah, MD

BM Zeeshan Hameed, MD

zeeshanhameedbm@gmail.com

Department of Urology, Kasturba Medical College Manipal, Manipal Academy

of Higher Education, Manipal (India)

Patricia Zondervan, MD

Academic Medical Center, University of Amsterdam, Netherlands

Jean McDonald, MD

North Middlesex University Hospital, Sterling Way, London (UK)

Bhavan Prasad Rai, MD

Department of Urology, Freeman Hospital, Newcastle upon Tyne NE7 7DN (UK)

Hadis Karimi, $M D$

Manipal College of Pharmacy, Manipal Academy of Higher Education, Manipal, India

Joanne Cresswell, MD

The James Cook University Hospital, South Tees Trust, Middlesbrough, United Kingdom

BM Zeeshan Hameed, Professor (Corresponding Author)

zeeshanhameedbm@gmail.com

Department of Urology, Kasturba Medical College, Manipal, Manipal Academy

of Higher Education, Manipal, India - 576104 